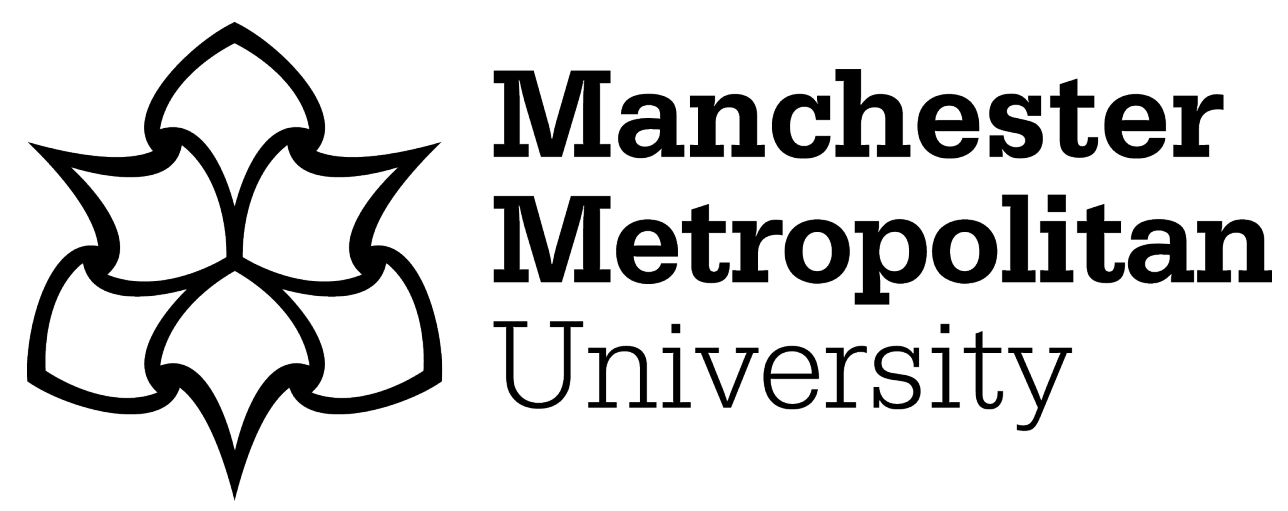

Bonat, WH, Olivero, J, Grande-Vega, M, Farfán, MA and Fa, John (2017) Modelling the covariance structure in marginal multivariate count models: Hunting in Bioko Island. Journal of Agricultural, Biological, and Environmental Statistics, 22 (4). pp. 446-464. ISSN 1085-7117

Downloaded from: https://e-space.mmu.ac.uk/618467/

Version: Accepted Version

Publisher: Springer Verlag

DOI: https://doi.org/10.1007/s13253-017-0284-7

Please cite the published version 


\title{
Modelling the covariance structure in marginal multivariate count models: Hunting in Bioko Island
}

\author{
W. H. Bonat* and J. Olivero ${ }^{\dagger}$ and M. Grande-Vega ${ }^{\ddagger}$ \\ and M. A. Farfán` and J. E. Faף
}

August 22, 2016

\begin{abstract}
We present a flexible statistical modelling framework to deal with multivariate count data along with longitudinal and repeated measures structures. The covariance structure for each response variable is defined in terms of a covariance link function combined with a matrix linear predictor involving known matrices. To specify the joint covariance matrix for the multivariate response vector the generalized Kronecker product is employed. The count nature of the data is taken into account by means of the power dispersion function associated with the Poisson-Tweedie distribution. Furthermore, the score information criterion is extended for selecting the components of the matrix linear predictor. We analyse a dataset consisting of prey animals (the main hunted species, the blue duiker Philantomba monticola and other taxa)
\end{abstract}

\footnotetext{
*Department of Mathematics and Computer Science, University of Southern Denmark, Odense, Denmark. Department of Statistics, Paraná Federal University, Centro Politécnico, Curitiba 81531980, CP 19081, Paraná, Brazil. E-mail: wbonat@ufpr.br

†niversidad de Málaga, Grupo de Biogeografía, Diversidad y Conservación, Departamento de Biologia Animal, Facultad de Ciencias, Campus de Teatinos s/n, 29071 Málaga, Spain.

${ }^{\ddagger}$ Research Group for Sustainable Management Silvanet, Faculty of Forestry, Technical University of Madrid, Ciudad Universitaria, 28040 Madrid, Spain and Asociación Ecotono, Paseo de la Habana 109. 2 A 28036 Madrid, Spain.

§Universidad de Málaga, Grupo de Biogeografía, Diversidad y Conservación, Departamento de Biología Animal, Facultad de Ciencias, Campus de Teatinos s/n, 29071 Málaga, Spain and BioGea Consultores, C/Navarro Ledesma 243, Portal 4-3 C, 29010 Málaga, Spain.

IDivision of Biology and Conservation Ecology, School of Science and the Environment, Manchester Metropolitan University, Manchester M1 5GD, UK and Center for International Forestry Research (CIFOR), CIFOR Headquarters, Bogor 16115, Indonesia.
} 
shot or snared for bushmeat by 52 commercial hunters over a 33-month period in Pico Basilé, Bioko Island, Equatorial Guinea. By taking into account the severely unbalanced repeated measures and longitudinal structures induced by the hunters and a set of potential covariates (which in turn affect the mean and covariance structures), our method can be used to indicate whether there was statistical evidence of a decline in blue duikers and other species hunted during the study period. Determining whether observed drops in the number of animals hunted are indeed true is crucial to assess whether species depletion effects are taking place in exploited areas anywhere in the world. We suggest that our method can be used to more accurately understand the trajectories of animals hunted for commercial or subsistence purposes, and establish clear policies to ensure sustainable hunting practices.

Keywords: Multivariate models; Estimating functions; Hunting; Longitudinal data

\section{Introduction}

Multivariate regression models have been of increased interest in the statistical literature. Recent applications include functional disability data (Manrique-Vallier; 2014), cognitive functioning (Anderlucci and Viroli; 2015), evolutionary biology (Cybis et al.; 2015), multi-species distribution (Hui et al.; 2015; Ovaskainen and Soininen; 2011), social, economic (Klein, Kneib, Lang and Sohn; 2015; Klein, Kneib, Klasen and Lang; 2015) and political sciences (Lagona et al.; 2015) to cite a few.

The mentioned methodologies apply latent variables or finite mixture of regression models to describe the covariance structure introduced by the multiple response variables. In contrast to these approaches Bonat and Jørgensen (2016) proposed the multivariate covariance generalized linear models (McGLMs), which explicitly model the marginal covariance matrix combining a covariance link function and a matrix linear predictor composed of known matrices. McGLMs have much in common with the GEE (Generalized Estimating Equations) (Liang and Zeger; 1986) approach popular in the analysis of longitudinal data. However, McGLMs were explicitly designed to deal with multiple response variables and allow for a flexible modelling of the covariance structure. On the other hand, current GEE implementations (Højsgaard et al.; 2006) deal only with one response variable and include a short list of pre-specified covariance structures, such as autoregression and compound symmetry.

Generalized linear mixed models (GLMMs) (Breslow and Clayton; 1993) are flexible models for handling multivariate data (Verbeke et al.; 2014). GLMMs are com- 
putationally demanding, and many algorithms have been proposed in the past three decades, see McCulloch (1997) and Fong et al. (2010) for reviews and further references. Rodrigues-Motta et al. (2013) presented a specific example of GLMM for count data. An aspect of GLMMs that gives rise to concern is the general lack of a closed-form expression for the likelihood and the marginal distribution of the data vector. A related question is the special interpretation of parameters inherent from the construction of GLMMs. Thus, the covariate effects are conditional on the latent variables, whereas the correlation structure is marginal for the latent variables rather than for the response variables.

The multivariate Poisson (Tsionas; 1999) and negative binomial (Shi and Valdez; 2014) distributions are suitable approaches to deal with multivariate count data. The multivariate Poisson has the restriction to deal only with equidispersed and positive correlated data. The last restriction is also shared by the multivariate negative binomial model. The assumption of a common error distribution required for these models may not be satisfied in practice, and methods for handling the case of unequal marginal distributions do not seem easily available. Additional methods for specifying models for dependent data include the Gaussian copula marginal regression models (Masarotto and Varin; 2012) and the class of hierarchical generalized linear models (Lee and Nelder; 1996).

In the context of multivariate longitudinal models, besides the modelling of the covariance structure between response variables, we also have to model the longitudinal and repeated measures structures for each response variable, i.e. the within covariance structure. The question of how to model the within covariance structure in the univariate case is often solved by choosing from a short list of options, such as compound symmetry, autoregressive and unstructured (Diggle et al.; 2002). Such choices are, however, not suitable for the combination of multivariate, repeated measures and longitudinal structures found in the application described in the Section 2. It motivates the development of a more general and flexible approach for covariance modelling in multivariate longitudinal count models.

In this paper, we adopt the McGLM framework in order to present a multivari- ate model suitable to deal with count response variables. Our model also relies on the structure of the multivariate discrete dispersion models (Jørgensen and Kokonendji; 2016), where the Poisson-Tweedie distribution provides a flexible framework for modelling discrete response variables. In this framework multivariate extensions of the Neyman Type A, Pólia-Aepply, negative binomial and Poisson-inverse Gaussian distributions appear as special cases. One advantage of this class of models is that similar to the exponential dispersion models (Jørgensen; 1997) the whole family is described by the power dispersion function, analogous to ordinary Tweedie exponential dispersion 
models with power variance functions. This fact allows us to specify models based on second-moment assumptions and use the engine of McGLMs for estimation and inference. For further references and regression models based on the Poisson-Tweedie distribution, see Bonat et al. (2016).

The model is motivated by a data set consisting of the number of blue duikers and other small animals shot or snared by 52 commercial hunters over a 33-month period in Pico Basilé, Bioko Island, Equatorial Guinea (Grande-Vega et al.; 2015). Bushmeat trade is an important resource in the livelihoods of many rural communities in West and central Africa. Overhunting for profit is known to cause immediate reductions in the density of targeted animals (Fa et al.; 2000). In extreme cases it may precipitate the disappearance of local populations and eventually result in the complete extirpation of a species (Fa and Brown; 2009). It is also known that hunted island animal populations are often at a greater risk of extinction because of their small geographic ranges and usually low population numbers (Grande-Vega et al.; 2015). In Bioko Island, the blue duiker (Philantomba monticola) is the most hunted species among 18 species of mammals and birds consumed as food.

The main goal of this data analysis is to investigate whether the number of hunted blue duikers declined during the study period. The data analysis should take into account the severely unbalanced repeated measures and longitudinal structures introduced by the hunters and a set of potential covariates affecting both the mean and covariance structures.

Determining whether the decline of hunted animals is instrumental, since it could suggest a reduction in the population of this species, with important applications for establishing policies of sustainable hunting practices. In this scenario, a bivariate count model is useful, since a significant negative correlation could indicate that hunters target another species as a result of the decline in the target species, while a non-significant correlation may push hunter to turn to alternative sources of income.

In view of the recent developments in the McGLMs framework the main contributions of this article are: i) introduces a suitable specification of the McGLMs to deal with the combination of longitudinal and repeated measures in the context of multivariate count data. ii) describes how to specify the components of the matrix linear predictor in order to take into account the effects of known covariates in a linear mixed model fashion. iii) extends the score information criterion (SIC) to select the components of the matrix linear predictor. iv) applied the methods to analyse the Hunting data set and $\mathrm{v}$ ) provides $\mathrm{R}$ code for constructing the components of the matrix linear predictor as well as fitting the models through the $\mathrm{mcg} I \mathrm{~m}$ (Bonat; 2016) package for the $\mathrm{R}$ statistical software.

We present the Hunting data set in Section 2. Section 3 discusses the model 
and its properties. We emphasize the specification of the matrix linear predictor. Section 4 extends the score information criterion for selecting the components of the matrix linear predictor. Section 5 describes the application of the model to the data. Section 6 discusses the main results. Finally, Section 7 presents the concluding remarks. The data set that is analysed in the paper and the programs that were used to analyse it can be obtained from

http ://www_leg-ufpr_br/doku_php/publications : papercompanions = hunting bioko2016.

\section{Data set}

The case study analysed in this paper uses data of animals hunted in the village of Basilé Fang, Bioko Norte Province, Bioko Island, Equatorial Guinea. The monthly number of blue duikers and other small animals shot or snared were collected from a random sample of 52 commercial hunters from August 2010 to September 2013. For each animal caught, the species, sex, method of capture and altitude were documented. The data set has 1216 observations. For additional description of the field work, see Grande-Vega et al. (2015).

In this analysis, we opted to aggregate the species into two levels blue duikers (BD) and other small animals (OT), since $\mathrm{BD}$ is the target species and OT are hunted at random. The covariates sex (Female, Male) and method (Firearm, Snare) are factors with two levels. The covariate alt is a factor with 5 levels $(300-600,601-900,901-$ $-1200,1201-1500$ and $>1500$ ) indicating the altitude where the animal was caught. Finally, the number of hunter days per month was recorded. It is important, because represents the effort employed by the hunter and should be used as an offset(in logarithm scale) for modelling the counts of hunted animals.

The study design introduces some sources of dependence in the data. We call hunter-month the effect of all observations taken at the same hunter and month. The hunter effect is represented by all observations taken at the same hunter. The Iongitudinal effect is introduced by the observations taken at sequentially months. The within covariance for each outcome can also be affected by the covariates in a linear mixed model fashion, see Section 3 and Demidenko (2013) for details. Finally, the correlation between response variables should be taken into account, since it plays an important role in terms of model interpretation. The number of observations per hunter-month and hunters varied between 1 and 16 and 1 and 104, respectively. These numbers show the severely unbalanced repeated measures and longitudinal structures present in the data set. 

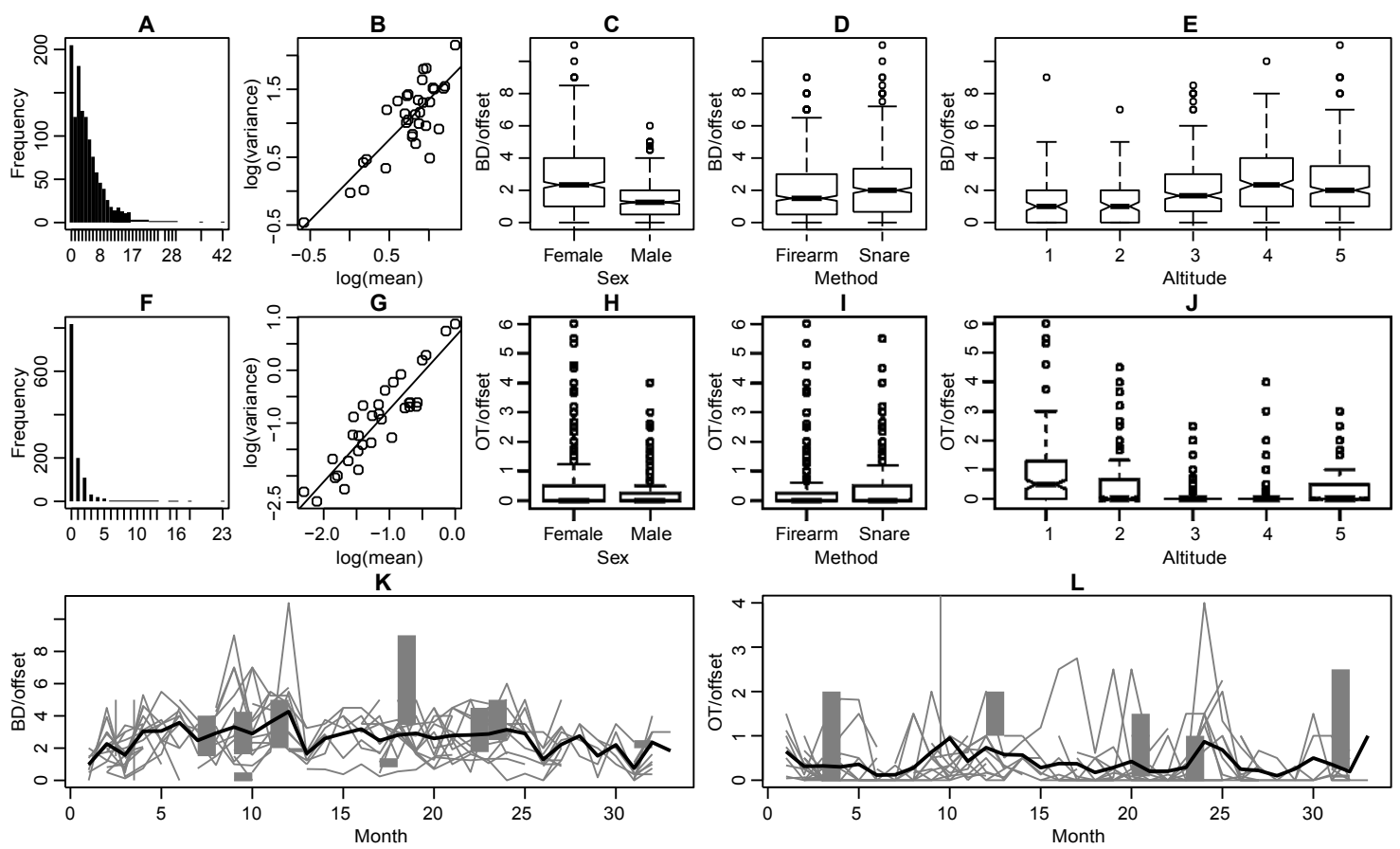

Figure 1: Histograms ( $A$ and $F$ ). Taylor plot (hunter mean and variance in double logarithmic scale) (B and $G)$. Boxplots for sex ( $C$ and $H)$, method (D and I) and a It ( $\mathrm{E}$ and $\mathrm{J}$ ). Individual average (gray) and overall average (black) trajectories ( $\mathrm{K}$ and $L$ ) for $B D$ and $O T$, respectively.

Histograms in Figure 1 suggest that the two error distributions may not be identical, and hint at potential problems with excess of zeroes and overdispersion. Boxplots suggest an effect of all covariates, whereas the approximate linearity of the Taylor plots suggest a variance function of power form.

\section{Multivariate longitudinal models for count data}

Let $Y_{N \times R}=\left\{Y_{1}, \ldots, Y_{R}\right\}$ be a response variable matrix and let $M_{N \times R}=\left\{\mu_{1}, \ldots, \mu_{R}\right\}$ denote the corresponding matrix of expected values. Let $\Sigma_{r}$ denote the $\mathrm{N} \times \mathrm{N}$ covariance matrix within the response variable $r$ for $r=1, \ldots, R$. Similarly, let $\Sigma_{b}$ be the $\mathrm{R} \times \mathrm{R}$ correlation matrix whose components $\rho_{r r}$ 's denote the correlation between the response variables $r$ and $r$ '. The multivariate covariance generalized linear model 
as proposed by Bonat and Jørgensen (2016) is given by

$$
\begin{aligned}
E(Y) & =M=\left\{g_{1}^{-1}\left(X_{1} \beta_{1}\right), \ldots, g_{R}^{-1}\left(X_{R} \beta_{R}\right)\right\} \\
\operatorname{Var}(Y) & =C=\Sigma_{R} \otimes \Sigma_{b}
\end{aligned}
$$

where $\Sigma_{R} \otimes \Sigma_{b}=\operatorname{Bdiag}\left(\tilde{\Sigma}_{1}, \ldots, \tilde{\Sigma}_{R}\right)\left(\Sigma_{b} \otimes I\right) \operatorname{Bdiag}\left(\tilde{\Sigma}_{1}^{\top}, \ldots, \tilde{\Sigma}_{R}^{\top}\right)$ is the generalized Kronecker product (Martinez-Beneito; 2013). The matrix $\tilde{\Sigma}_{r}$ denotes the lower tri-

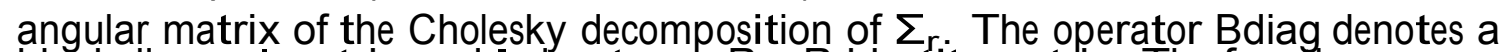
block diagonal matrix and $\mathbf{I}$ denotes an $\mathrm{R} \times \mathrm{R}$ identity matrix. The functions $\mathrm{g}_{\mathrm{r}}$ are link functions, for which we adopt the orthodox log-link function. Let $X_{r}$ denote an $\mathrm{N} \times \mathrm{k}_{\mathrm{r}}$ design matrix and $\beta_{\mathrm{r}} a \mathrm{k}_{\mathrm{r}} \times 1$ regression parameter vector. Note that, the model has a specific linear predictor for each response variable.

In order to specify the covariance within response variables, we adopt the definition of Jørgensen and Kokonendji (2016) for Poisson-Tweedie random vector, i.e.

$$
\Sigma_{r}=\operatorname{diag}\left(\mu_{r}\right)+V\left(\mu_{r} ; p_{r}\right)^{\frac{1}{2}}\left(\Omega\left(T_{r}\right)\right) V\left(\mu_{r} ; p_{r}\right)^{\frac{1}{2}}
$$

where $V\left(\mu_{r} ; p_{r}\right)=\operatorname{diag}\left(\mu_{r}^{p_{r}}\right)$, is a diagonal matrix whose main entries are given by the power variance function. This specification is a multivariate representation of the power dispersion function which characterizes the Poisson-Tweedie family, see Jørgensen and Kokonendji (2016) for details. Finally, following the ideas of Anderson (1973) and Pourahmadi (2000) we model the dispersion matrix $\Omega\left(\mathrm{T}_{\mathrm{r}}\right.$ ) as a linear combination of known matrices, i.e.

$$
\mathrm{h}\left(\Omega\left(\mathrm{T}_{\mathrm{r}}\right)\right)=\mathrm{T}_{\mathrm{r} 0} Z_{\mathrm{r} 0}+\cdots+\mathrm{T}_{\mathrm{rD}} Z_{\mathrm{rD}} .
$$

Here $h$ is the covariance link function, $Z_{r d}$ with $d=0, \ldots, D$ are known matrices reflecting the covariance structure within the response variable $r$, and $T_{r}=$ $\left(\mathrm{T}_{\mathrm{r} 0}, \ldots, \mathrm{T}_{\mathrm{rD}}\right)$ is $\mathrm{a}(\mathrm{D}+1) \times 1$ parameter vector. This structure is a natural analogue of the linear predictor of the mean structure, and following Bonat and Jørgensen (2016) we call it a matrix linear predictor.

In this paper we focus on the identity covariance link function, since many interesting models appear as special cases. Demidenko (2013) showed that the covariance structure induced by the orthodox Gaussian linear mixed model is a linear covariance matrix, i.e. has the form of (1). In this sense, the models presented in this paper can been seen as an extension of the Gaussian linear mixed model for handling count data. Furthermore, popular approaches to deal with longitudinal autocorrelated data, as the compound symmetry, moving average and first order autoregressive, are 
also covariance linear models. In what follows we discuss some of the possibilities for the specification of the matrix linear predictor in the context of longitudinal data.

Since the matrix linear predictor is specified for each response variable, suppose without loss of generality that $r=1$. Denote $y_{g o}$ an observation $o=1, \ldots, O_{g}$ within the group $g=1, \ldots, G$ and let $y_{g}$ denote the $O_{g}$-dimensional vector of measurements from the gth group. In particular, for the data set presented in Section 2 the groups are given by the Hunters. Thus, the response variable vector is given by $Y=$ $\left(y_{1}, \ldots, y_{G}\right)^{\top}$. Let $A_{g}$ denote an $O_{g} \times E$ design matrix composed of the values of $\mathrm{E}$ known covariates available to model the covariance structure. Furthermore, let $A_{g, e}$ denote the eth column of the matrix $A_{g}$. Following Demidenko (2013) the main effect of the covariate $e$ and the interaction effect between the covariates e and e' are included in the covariance model through the symmetric matrices

$$
A_{g}^{e}=A_{g, e} A_{g, e e}^{\top} \text { and } A_{g}^{e e^{\prime}}=A_{g, e} A_{g, e^{\prime}}^{\top}+A_{g, e^{e}} A_{g, e}^{\top} \text {, }
$$

respectively. The matrices $A_{g}^{e}$ and $A_{g}^{e e^{\prime}}$ are group specific. To obtain the components of the matrix linear predictor for the entire response variable vector $Y$, we assume independent groups. Thus, the components of the matrix linear predictor that measure the effect of the eth covariate and the interaction effect are given by

$$
Z_{e}=\operatorname{Bdiag}\left(A_{1}^{e}, \ldots, A_{G}^{e}\right) \text { and } Z_{e e^{\prime}}=\operatorname{Bdiag}\left(A_{1}^{e e^{\prime}}, \ldots, A_{G}^{e e^{\prime}}\right) \text {, }
$$

where as before the operator Bdiag denotes a block diagonal matrix. The matrices $Z_{e}$ and $Z_{e e}$ ' can be included as the $Z_{d}$ 's components in the matrix linear predictor, see 1. When the main and interaction effects are included in the model, we have $E(E+1) / 2$ components. A simplification is obtained by considering only main effects resulting in $E$ components. In general, we reserve the first component of the matrix linear predictor $Z_{0}$ to an identity matrix, that represents the intercept of the linear covariance model.

Demidenko (2013) showed that some well known covariance structures used to model longitudinal and repeated measures data are linear covariance models. To describe these structures consider a particular group g with three observations. As before to extend the matrices to the entire response variable vector, we assume independent groups and use the Bdiag operator. The compound symmetry or exchangeable structure is a linear combination of an identity and a matrix of ones, i.e. for this particular group the matrix linear predictor is given by

$$
\Omega_{\mathrm{g}}(\mathbf{T})=\mathrm{T}_{0} \square \begin{array}{rllrll}
1 & 0 & 0 & 1 & 1 & 1 \\
1 & 0 \square & \mathrm{T}_{1} \square & 1 & 1 & 1 \\
0 & 0 & 1 & 1 & 1 & 1
\end{array} .
$$


The Moving Average model of order $p M A(p)$ is also a linear covariance model. The components of the matrix linear predictor associated with the MA(1) and MA(2) structures are given respectively by

$$
\mathrm{A}_{1}=\begin{array}{rll}
\square & 1 & 0 \\
& 0 & 1
\end{array} \quad \text { and } \quad \mathrm{A}_{2}=\begin{array}{rll}
\square & 0 & 1 \\
0 & 1 & 0
\end{array} \quad \begin{array}{rll}
0 & 0 \\
1 & 0 & 0
\end{array}
$$

For longitudinal data analysis, we can use the inverse of Euclidean distance between pairs of observations as a component of the matrix linear predictor, for example

$$
\mathrm{A}_{1}=\begin{array}{ccc}
\square & 1 / \mathrm{d}_{12} & 1 / \mathrm{d}_{13} \\
\square 1 / \mathrm{d}_{12} & 0 & 1 / \mathrm{d}_{23} \square, \\
1 / \mathrm{d}_{13} & 1 / \mathrm{d}_{23} & 0
\end{array}
$$

where $d_{i j}$ denotes the Euclidean distances between the observations at time $\mathbf{i}$ and j. By combining the simple structures described above, we have a flexible set of components to compose the matrix linear predictor for the analysis of longitudinal data. Demidenko (2013) also showed that the popular first-order autoregression model can be written as a linear covariance model, but using the inverse covariance link function. In this paper, we do not pursue in this covariance link function.

The power parameter $\mathrm{p}$ plays an important role in the context of multivariate Poisson-Tweedie models, since it is an index which distinguishes between some important discrete distributions. Examples include the Neyman Type A $(p=1)$, PólyaAeppli $(p=1.5)$, negative binomial $(p=2)$ and Poisson-inverse Gaussian $(p=3)$. The algorithm proposed by Bonat and Jørgensen (2016) allows us to estimate the power parameter, which works as an automatic distribution selection.

\section{The score information criterion}

In this section, we extend the score information criterion (SIC) proposed by Stoklosa et al. (2014) for the selection of the components of the matrix linear predictor. In order to introduce the SIC, we first present some key components of the estimating function approach used to fit McGLMs. The algorithm and asymptotic theory associated with the estimating function estimators were presented by Bonat and Jørgensen (2016) and implemented in the $\mathrm{mcg} I \mathrm{~m}$ (Bonat; 2016) package for the $\mathrm{R}$ ( $\mathrm{R}$ Core Team; 2015) statistical sofware.

The second-moment assumptions of McGLMs motivate us to divide the set of parameters into two subsets $\theta=\left(\beta^{\top}, \lambda^{\top}\right)^{\top}$. In this notation $\beta=\left(\beta_{1}^{\top}, \ldots, \beta_{R}^{\top}\right)^{\top}$ 
and $\lambda=\left(\rho_{1}, \ldots, \rho_{R(R-1) / 2}, p_{1}, \ldots, p_{R}, \mathbf{T}_{1}^{\top}, \ldots, \mathbf{T}_{R}^{\top}\right)^{\top}$ denote a $\mathrm{K} \times 1$ and $\mathrm{Q} \times 1$ vector of all regression and dispersion parameters, respectively. Let $Y=\left(Y_{1}^{\top}, \ldots, Y_{R}^{\top}\right)^{\top}$ and $M=\left(\mu_{1}^{\top}, \ldots, \mu_{R}^{\top}\right)^{\top}$ denote the $N R \times 1$ stacked vector of the response variable matrix $Y_{N \times R}$ and expected values matrix $M_{N \times R}$ by columns, respectively.

The regression coefficients are estimated by using the orthodox quasi-score function (Bonat and Jørgensen; 2016; Liang and Zeger; 1986). The dispersion parameters are estimated based on the Pearson estimating function, defined by the components

$$
\psi_{\lambda_{i}}(\beta, \lambda)=\operatorname{tr}\left(W_{\lambda_{i}}\left(r^{\top} r-C\right)\right) \quad \text { for } \quad \mathbf{i}=1, \ldots, Q,
$$

where $\mathrm{W}_{\lambda_{i}}=-\partial \mathrm{C}^{-1} / \partial \lambda_{i}$ and $r=\mathrm{Y}-\mathrm{M}$.

Two key components of an estimating function approach are the sensitivity and variability matrices. The entry $(\mathbf{i}, \mathbf{j})$ of the $Q \times Q$ sensitivity matrix of $\psi_{\lambda}$ is given by,

$$
S_{\lambda_{i j}}=E \frac{\partial}{\partial \lambda_{i}} \psi_{\lambda_{j}}=-\operatorname{tr} W_{\lambda_{i}} C W_{\lambda_{j}} C \text {. }
$$

Similarly, the entry $(\mathbf{i}, \mathbf{j})$ of the $Q \times Q$ variability matrix of $\psi_{\lambda}$ is given by

$$
V_{\lambda_{i j}}=\operatorname{Cov}\left(\Psi_{\lambda_{i}}, \Psi_{\lambda_{j}}\right)=2 \operatorname{tr}\left(W_{\lambda_{i}} C W_{\lambda_{j}} C\right)+k_{I=1}^{N R} k_{I}^{(4)}\left(W_{\lambda_{i}}\right)_{I I}\left(W_{\lambda_{j}}\right)_{I I}
$$

where $k_{1}^{(4)}$ denotes the fourth cumulant of $Y_{1}$. In order to keep the model based on second-moment assumptions only, we following Bonat and Jørgensen (2016) use the empirical fourth cumulant.

Stoklosa et al. (2014) in the context of generalized estimating equations (GEE) proposed the score information criterion (SIC) to be used with forward selection algorithms in the cases where we have a large number of covariates to compose the linear predictor. The SIC is based on the score statistics, what becoming such criterion convenient, since it can be computed for all candidate models without actually fitting them.

Suppose without loss of generality that $r=1$ and fixed power parameter. In that case, the vector of dispersion parameters simplify to $\lambda=\mathrm{T}$, since we have no correlation neither power parameters. For a given mean structure, suppose that the parameter vector $\mathbf{T}$ can be partitioned as $\mathbf{T}=\left(\mathbf{T}_{1}^{\top}, \mathbf{T}_{2}^{\top}\right)^{\top}$, whose dimension are $(\mathrm{Q}-\mathbf{s}) \times 1$ and $s \times 1$, respectively. The Pearson estimating function $\psi_{\lambda}$ and its sensitivity and variability matrices, can also be partitioned to $\psi_{\lambda}(\beta, \mathbf{T})=\left(\psi_{\lambda_{1}}\left(\beta, \mathbf{T}_{1}\right)^{\top}, \Psi_{\lambda_{2}}\left(\beta, \mathbf{T}_{2}\right)^{\top}\right)^{\top}$,

$$
S_{\lambda}=\begin{array}{ll}
S_{\lambda_{11}} & S_{\lambda_{12}} \\
S_{\lambda_{21}} & S_{\lambda_{22}}
\end{array}
$$


and

$$
\mathrm{V}_{\lambda}=\begin{array}{ll}
\mathrm{V}_{\lambda_{11}} & \mathrm{~V}_{\lambda_{12}} \\
\mathrm{~V}_{\lambda_{21}} & \mathrm{~V}_{\lambda_{22}}
\end{array}
$$

respectively. The null hypothesis $\mathrm{H}_{0}$ is $\mathrm{T}_{2}=0$. Let $\widetilde{\mathbf{T}}=\left(\hat{\mathrm{T}}_{1}^{\top}, 0^{\top}\right)^{\top}$ be the vector of Pearson estimates under $\mathrm{H}_{0}$. Note that, only the base model containing $\hat{\mathbf{T}}_{1}$ parameters has to be fitted. In practical situations, this model can contain only a simple intercept. The Pearson estimating function takes the form

$$
\psi_{\lambda}(\beta, \widetilde{\mathrm{T}})=\left(\psi_{\lambda_{1}}^{\top}(\beta, \widetilde{\mathrm{T}}), \psi_{\lambda_{2}}^{\top}(\beta, \widetilde{\mathrm{T}})\right)^{\top}=\left(0^{\top}, \psi_{\lambda_{2}}^{\top}(\beta, \widetilde{\mathrm{T}})\right)^{\top} .
$$

The generalized score statistic is given by

$$
\mathrm{T}_{\lambda_{2}}(\beta, \widetilde{\mathbf{T}})=\psi_{\lambda_{2}}^{\top}(\beta, \widetilde{\mathbf{T}}) \operatorname{Var}\left(\psi_{\lambda_{2}}(\beta, \widetilde{\mathbf{T}})\right)^{-1} \psi_{\lambda_{2}}(\beta, \widetilde{\mathbf{T}})
$$

where

$$
\begin{aligned}
\operatorname{Var}\left(\psi_{\lambda_{2}}(\beta, \widetilde{\mathrm{T}})\right) & =\mathrm{V}_{\lambda_{22}}-\mathrm{S}_{\lambda_{21}} S_{\lambda_{11}}^{-1} \mathrm{~V}_{\lambda_{12}}-\mathrm{V}_{\lambda_{12}} S_{\lambda_{11}}^{-1} S_{\lambda_{12}} \\
& +S_{\lambda_{21}} S_{\lambda_{11}}^{-1} V_{\lambda_{11}} S_{\lambda_{11}}^{-1} S_{\lambda_{12}}
\end{aligned}
$$

is the variance of the subvector $\psi_{\lambda_{2}}(\beta, \widetilde{\mathbf{T}})$. Under the null hypothesis, $T_{\lambda_{2}}(\beta, \widetilde{\mathbf{T}})$ has a chi-square distribution with $s$ degrees of freedom. In practice, all quantities in (5) are evaluated at the Pearson estimates under the null hypotheses. If $\mathrm{H}_{0}$ were true, then $\psi_{\lambda_{2}}(\beta, \widetilde{\mathbf{T}})$ that is the Pearson estimating function for $\mathbf{T}_{2}$ would be close to zero when evaluated under the null. Large values of $T_{\lambda_{2}}(\beta, \widetilde{\mathrm{T}})$ would argue against $\mathrm{H}_{0}$. The main idea behind SIC is to use (5) as a quadratic approximation to the log-likelihood ratio statistic. The so-call one-step SIC is defined by

$$
\operatorname{sIC}^{(1)}(\beta, \mathbf{T})=-T_{\lambda_{2}}(\beta, \widetilde{\mathbf{T}})+\delta|\mathbf{T}|
$$

Note that this criterion is a function of $\widetilde{\mathrm{T}}$ only, thus only the base model needs to be fitted. As point out by Stoklosa et al. (2014) the approximation of score statistics to likelihood ratio statistics can be poor when there is a significant departure from the null model. Hence an improved approximation might calculate the score statistic in one-parameter increments, i.e.

$$
\operatorname{SIC}(\beta, \mathbf{T})=-\max _{s_{s=1} T_{(s)} \in T_{2}^{\prime s-1}}\left\{T_{\lambda_{2}(s)}\left(\beta, \widetilde{T}_{s-1}\right)\right\}+\delta|\mathbf{T}|
$$

where $\mathbf{T}_{\mathrm{s}}^{\top}=\left(\mathbf{T}_{\mathrm{s}-1}^{\top}, \mathbf{T}_{\mathrm{s}}\right)$ and $\mathbf{T}_{2}^{\sharp \mathrm{s}-1}=\mathbf{T}_{2} \cap \mathbf{T}_{\mathrm{s}-1}^{\mathrm{c}}$ where $\mathbf{T}_{\mathrm{s}-1}^{\mathrm{c}}$ is the complement set of $\mathbf{T}_{\mathbf{s}-1}$. In summary, we sequentially add new parameters selected from $\mathbf{T}_{2}$, these are $\mathrm{T}_{(\mathrm{s})}$ for $\mathrm{s}=1, \ldots,\left|\mathrm{T}_{2}\right|$, in the order that maximizes the score statistic (5) in each step. 
In that case no more than $\left|\mathbf{T}_{2}\right|$ models will be fitted to reach the final model. In this paper we consider the penalties $\delta=2$, as it is analogous to the Akaike information criterion. It is also possible to use $\delta=\log N$ to have an analogous to the Bayesian information criterion.

\section{Results}

In this section, we apply the McGLM for multivariate count data to analyse the data set presented in Section 2. The second-moment assumptions of the McGLM require the specification of a linear predictor and a matrix linear predictor for each response variable. In this application, for composing the linear predictor we have three covariates sex, method and alt along with the time trend month. We considered interaction terms up to second order between the four main effects. The time trend was modelled as a polynomial of third and fourth degrees for BD and OT, respectively. Such choices were based on exploratory analysis and preliminary fits as we shall explain better in the Section 6 . In all fitted models the number of hunter days (in logarithm scale) was used as an offset.

To specify the matrix linear predictor, we have the repeated measures structures represented by the Hunter and Hunter-Month effects. The Longitudinal effect introduced by the observations taken at sequentially months and the three covariates, sex, method and alt. For the repeated measures effects we assumed a compound symmetry (of ones) structure,see (3). The longitudinal effect was modelled using the inverse of Euclidean distances,see (4). Finally, the covariates are included in the covariance model in a linear mixed model fashion, see 2. In this application for model parsimony and since we have only categorical covariates to compose the matrix linear predictor, we considered only main effects.

For clarity, consider a particular Hunter that represents the group structure described in the Section 3. Furthermore, consider that we have four observations (two for the first month and two for the second month). Consider also for simplicity that we have the values of a covariate $e=\left(e_{1}, e_{2}, e_{3}, e_{4}\right)$. In that case, the matrix linear predictor has the following form 


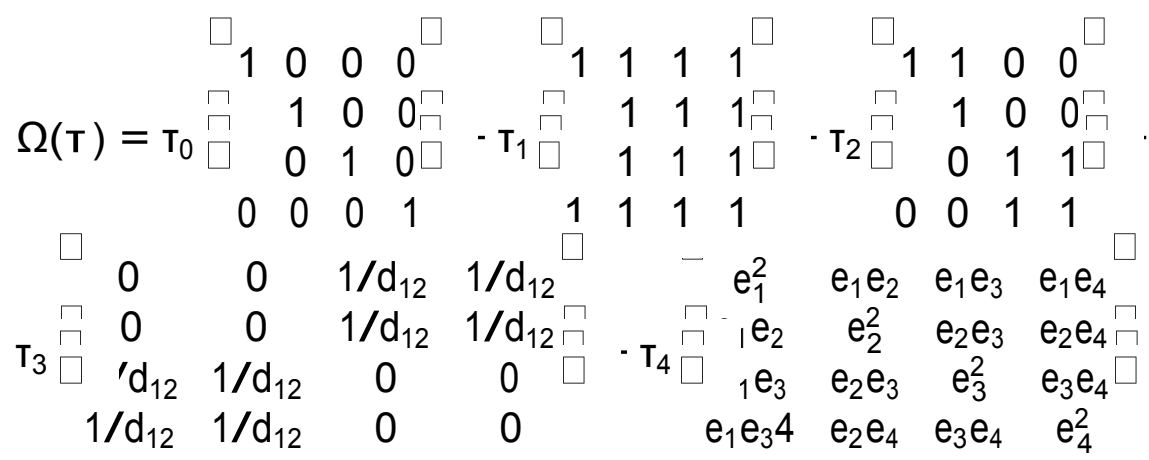

where $T_{0}$ is the intercept of the covariance linear model. The parameters $T_{1}, T_{2}$, $\mathrm{T}_{3}$ and $\mathrm{T}_{4}$ measure the Hunter, Hunter-Month, Longitudinal and covariate effects, respectively.

We employed a stepwise procedure for selecting the components of the linear and matrix linear predictors. The SIC using penalty $\delta=2$ and the Wald test were used in the forward and backward steps, respectively. We defined as stop criterion SIC $>0$, since in that case the penalty is larger than the score statistics.

Our strategy to select the final model consists of: i) select the components of the linear predictor for each response variable fixing the covariance structure assuming independent observations, i.e. $Z_{0}=\mathrm{I}$. ii) select the components of the matrix linear predictor for each response variable fixing the mean structure obtained in step (i). iii) fit the multivariate model and iv) remove non-significant effects in both linear and matrix linear predictors if any. In this application after fit the multivariate model all covariates selected to compose the linear and matrix linear predictors were significant. Supplemenaty Tables S1 and S2 present the step-by-step procedure. Table 1 presents the Wald statistics for the components of the selected linear predictor for each response variable obtained by fitting the final multivariate model. The selected matrix linear predictors were composed of a diagonal matrix (Intercept) combined with the Hunter-Month, Method and Longitudinal effects for BD and only the Hunter-Month effect for OT.

The results in Table 1 show that the method effect for the response variable oт was non-significant, but given its highly significant interaction with alt we opted to keep this effect in the model. Table 2 shows the estimates, standard errors (SE) and Z-statistics for the power and dispersion parameters for the final model.

The estimates of the power parameters suggest that the Neyman Type $A(p=1)$, which indicates a zero inflation relative to the Poisson distribution is a suitable choice for both response variables. For the response variable oт the Pólya-Aeppli $(p=1.5)$ can also be suggested. The correlation between response variables was weak -0.0532 
Table 1: Wald statistics $\left(x^{2}\right)$, degrees of freedom (Df) and p-values for the components of the selected linear predictor for each response variable.

\begin{tabular}{lccc|lccc}
\hline & \multicolumn{2}{c}{ BD } & \multicolumn{5}{c}{ OT } \\
\cline { 5 - 7 } Effects & Df & $\mathrm{X}^{2}$ & $\mathrm{p}$-value & Effects & $\mathrm{Df}$ & $\mathrm{X}^{2}$ & p-value \\
\hline method & 1 & 6.986 & 0.008 & method & 1 & 1.766 & 0.183 \\
alt & 4 & 138.262 & 0.000 & alt & 4 & 128.042 & 0.000 \\
sex & 1 & 247.843 & 0.000 & sex & 1 & 15.927 & 0.000 \\
month & 3 & 25.791 & 0.000 & month & 4 & 10.150 & 0.038 \\
method:alt & 4 & 58.688 & 0.000 & method:alt & 4 & 26.455 & 0.000 \\
alt :month & 12 & 43.898 & 0.000 & alt:sex & 4 & 13.238 & 0.012 \\
- & - & - & - & alt:month & 16 & 90.365 & 0.000 \\
\hline
\end{tabular}

Table 2: Power and dispersion parameter estimates, standard errors (SE) and Zstatistics for the components of the selected matrix linear predictor for each response variable.

\begin{tabular}{lccc|ccc}
\hline & \multicolumn{3}{c}{$\mathrm{BD}$} & \multicolumn{3}{c}{$\mathrm{OT}$} \\
\hline Effects & Estimate & $\mathrm{SE}$ & Z-statistics & Estimate & $\mathrm{SE}$ & Z-statistics \\
\hline power & 1.165 & 0.115 & 10.108 & 1.453 & 0.251 & 5.777 \\
Intercept & 0.474 & 0.142 & 3.345 & 0.686 & 0.184 & 3.737 \\
Hunter-Month & 0.722 & 0.151 & 4.792 & 0.294 & 0.093 & 3.163 \\
Method & 0.928 & 0.258 & 3.603 & - & - & - \\
Longitudinal & -0.155 & 0.0424 & -3.660 & - & - & - \\
\hline
\end{tabular}


(0.0287) and not significantly different from 0 .

It is interesting to highlight that the $\Omega$ matrix describes the part of the covariance structure that does not depend on the mean structure. Thus, it is interesting to interpret the parameters that compose this matrix in terms of the correlation introduced by its components. For example, the correlation introduced by the Hunter-Month effect is $0.604(0.0594)$ and $0.299(0.102)$ for BD and OT, respectively. These numbers are easily obtained by $\hat{\mathrm{T}}_{1} /\left(\hat{\mathrm{T}}_{0}+\hat{\mathrm{T}}_{1}\right)$. Similarly, the correlation between observations taken at the same hunter by the method snare is $0.652(0.074)$. Note that, since the Hunter effect was not significant the reference level is the Intercept i.e. independence. Thus, we have no evidence of dependence between observations taken at the same hunter by the method firearm. Finally, the correlation introduced by the Longitudina I effect is $-0.487(0.203)$ for lag equals 1 . The numbers in the brackets denote the standard error computed using the delta method.

Figures 2 and 3 present the fitted values and 95\% confidence intervals for the response variables $\mathrm{BD}$ and $\mathrm{OT}$, respectively. We plot the observed values divided by the offset and the fitted values were computed fixing the offset equals 1 . Supplementary Tables S3 and S4 present the estimates and standard errors for the regression coefficients associated with the response variables BD and OT, respectively.

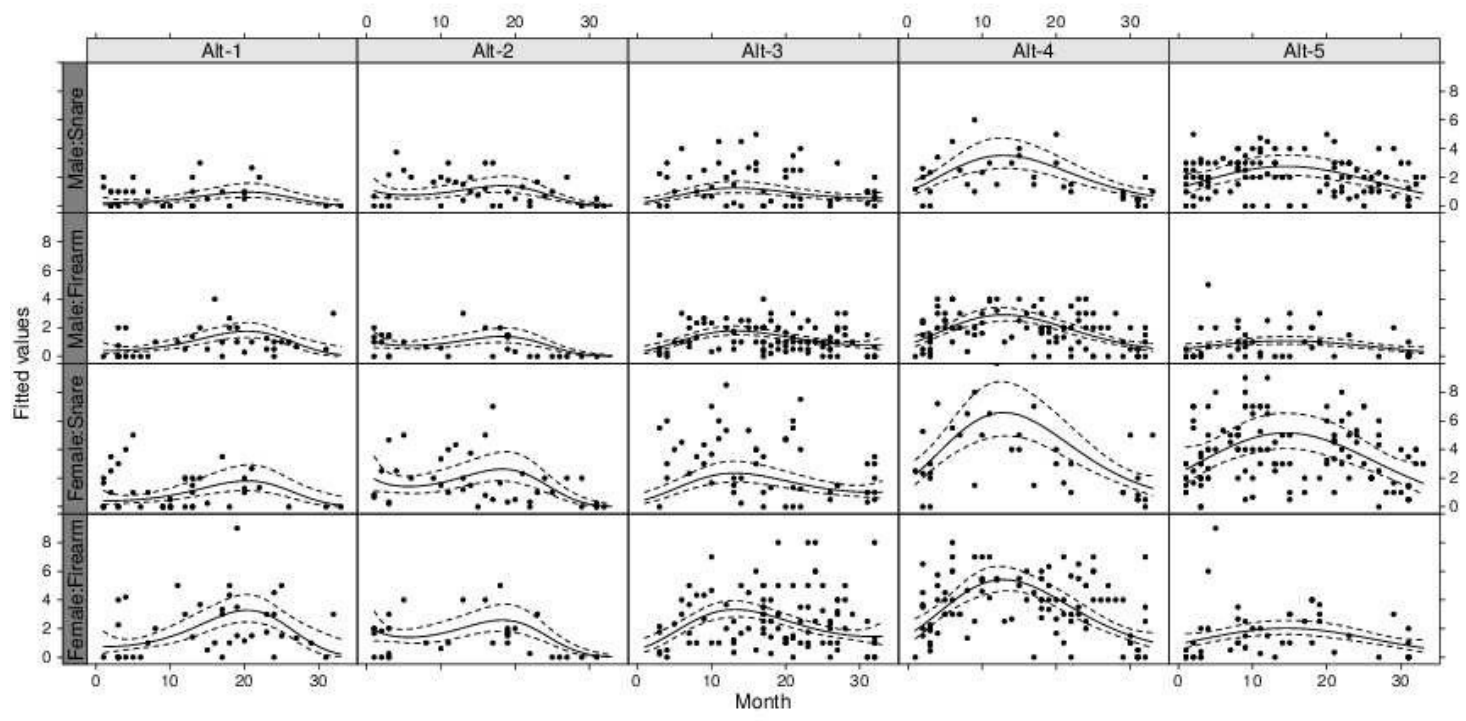

Figure 2: Fitted values and $95 \%$ confidence intervals by altitude, method of capture and sex for the response variable $\mathrm{BD}$.

Figure 2 shows that for all altitudes the number of hunted blue duikers increases 


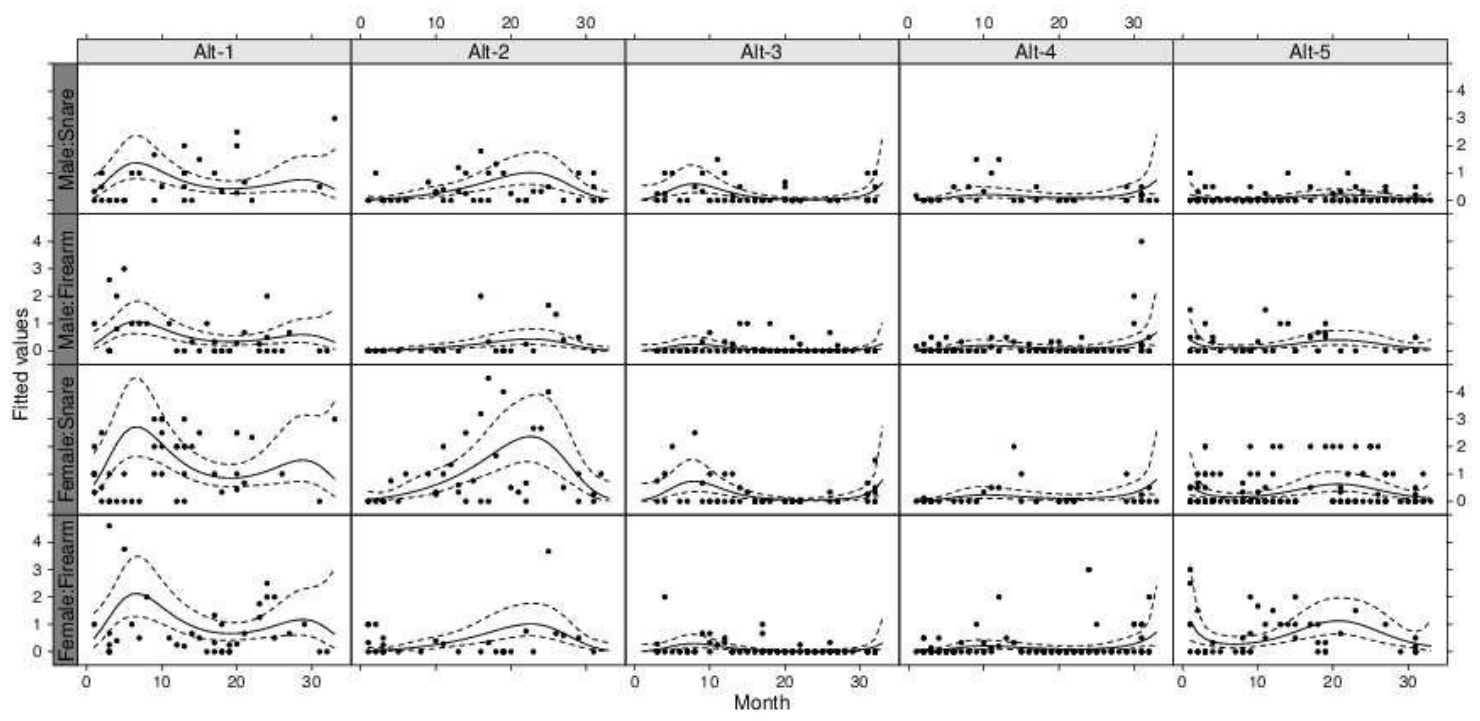

Figure 3: Fitted values and $95 \%$ confidence intervals by altitude, method of capture and sex for the response variable от.

from the beginning to the middle of the data collection, when a clear decreases start with sensible differences in the threshold point among the levels of the covariate alt. Altitudes 4 and 5 present the largest numbers of caught animals while altitudes 1 and 2 the smallest ones.

Similar we have seen for BD Figure 3 shows a clear time trend for the response variable oт in the altitudes 1 and 2 . Altitudes 3 and 4 show a different pattern with a slightly increase at the end of the experiment. Altitudes 1 and 2 present the largest numbers of other animals hunted by both methods and sexes. The smallest numbers appear in altitudes 3 and 4 using firearms. In general the number of females hunted is bigger than males and the most effective method of capture depends on the altitude.

It is important to highlight that despite of the differences in terms of altitudes, sexes and methods seem small in its magnitude judging by the results presented in Figures 2 and 3 . Such impression is due to the fact that, such results were obtained by fixing the number of hunter days (offset) equals 1 . Thus, the differences tend to be amplified while the number of hunter days increases. Furthermore, the regression coefficients associated with these effects are in general significantly different from 0 (see Tables 1, S3 and S4). 


\section{Discussion}

This section discusses the results presented in Section 5. The main data analysis goal was to determine if there was evidence of depletion in the population of blue duikers and other small animals based on data of hunted animals. To detect such a depletion effect, we included in the model a special term that represented the time trend for which we allowed a flexible functional form through a polynomial of degree three and four for the response variables BD and ОT, respectively. To control other effects that were not of main interest, we included in the model the effects of covariates such as sex, method of capture and altitude. The irregular activity of the hunters introduces severely unbalanced repeated measures and longitudinal structures that were modelled through a matrix linear predictor composed of known matrices. Although these effects are not of main interest, they help us to understand the complex dynamics of hunting activity and provide us with insights of the general aspects of the population of the targeted taxa. In what follows we discuss the effect of all covariates.

The results presented in Section 5 showed that for both response variables (BD and От), methods (snare and firearm) and all altitudes, the number of females hunted was larger than males. Since hunters do not target any particular animal, this bias in sexes hunted could be a function of a greater hunting susceptibility of females or that there are more females in the population than males. With regards to the method of capture, our results showed that this covariate presents a highly significant interaction with the covariate altitude. For the response variable $\mathrm{BD}$ the regression coefficients presented in the Supplementary Table S3, show that the method firearm is the most effective in altitude 1 , while the method snare is the most effective in altitude 5 . For altitudes 2 to 4 the differences between the methods of capture are not significant. Regarding the response variable от the method snare is the most effective in altitudes 2 and 3 , while the method firearm is the most effective in altitude 5 . In the altitudes 1 and 4 there is no difference between the methods.

The covariate altitude reflected different hunting pressure at variable elevations in the study areas. Blue duikers may be overhunted in lower altitudes (1 and 2) because of the proximity to human settlements, which increases hunting pressure. It may explain why the number of blue duikers is lower in altitudes 1 and 2 . On the other hand, in altitudes 3 to 5 we presume that more animals are hunted because these areas are less exploited areas. The opposite situation appears for other small animals, this result may indicate a depletion effect. Often, when the bigger animals (such as blue duikers in Bioko) are hunted out, which may be happening in altitudes 1 and 2, smaller ones tend to increase in numbers. This phenomenon is known as 
density compensation (Fa and Brown; 2009).

While modelling the covariance structure we detected a significant effect of the covariate Hunter-Month for BD and От. This effect is clearly due to the way that the data were collected and the arbitrary monthly aggregation. For the response variable $\mathrm{BD}$ in addition to the Hunter-Month effect, the longitudinal structure showed a significant negative effect. This result indicates that hunters may be affecting the prey population. Hence, some time is required for the population to recover and may indicate overexploitation of the hunted blue duikers population. A strong correlation between observations taken by the method snare was detected, but none appeared between observations taken by the method firearm. Such result is expected since the use of firearms to hunt is more effective when killing larger animals, so we would expect that the number of prey to decline with hunting effort with guns. This effect was detected by the longitudinal effect. On the other hand, because the method snare requires a much more continuous effort, the observations are more similar and consequently correlated along the study period. This mix of methods of capture could explain that months with a large number of animals hunted were followed by months with a smaller number of animals taken, explaining the negative longitudinal effect detected.

Finally, the time trend showed that for the response variable BD the number of hunted animals increases from the beginning to the middle of the data collection, followed by an intense decline after that. The maximum number of animals hunted appeared around the months 20 and 14 for altitudes 1 to 2 and 3 to 5 , respectively. A possible explanation for this result could be that at the start of the study period the blue duiker population in the region were more numerous, but following intensive hunting the population starts to decrease and consequently the number of hunted animals also falls. Another explanation could be that there is interannual variation in numbers which may be related to changes in climate and by consequence productivity of the forest, but we have no additional data to confirm this hypothesis. The significant decline after the middle of the study period provides support for an overhunting effect.

The temporal pattern detected for the response variable oт is more volatile mainly in altitudes 1 and 2, indicating that the number of oт animals hunted could have been affected by many factors, including the availability of other species as well as economic and climate conditions. This volatile pattern may also explain the weak and non-significant correlation between OT and BD.

Modelling the time trend through a polynomial function was a data-driven decision based on exploratory analysis and preliminary fits. The preliminary fits consisted of fitting models using B-splines basis as implemented in the package splines for 
the R statistical software. To select the number of degrees of freedom required for the B-splines basis, we fitted models using different degrees of freedom and check the significance of their regression coefficients using Wald test. Based on this procedure, we obtained that for the response variables ВТ and от three and four degrees of freedom were enough to provide a suitable fit. Furthermore, based on the behaviour of the fitted values and given the low number of degrees of freedom required by the B-spline basis, we detected that a simple polynomial could provide a suitable fit. Thus, we fitted the model changing the B-spline basis by polynomial of three and four degrees of freedom for the response variables BT and OT, respectively.

We compared the fitted model with the one obtained by using the B-spline basis in terms of Gaussian pseudo-likelihood (GPL) (Carey and Wang; 2011). GPL is a measure similar to the log-likelihood value in the context of maximum likelihood estimation. Thus, larger values indicate better fit. The value of the GPL for the model presented in the Section 5 was -4463.330 . Similarly, the value of the GPL for the model fitted using the B-splines basis was -4462.270 . The GPL indicated that the fits are quite similar. Furthermore, we also compared the fitted values obtained from both models that were virtually the same. Thus, we opted to present the model fitted using the polynomial. The advantage of the polynomial is that it is more familiar to applied researchers than the B-spline basis.

To provide more sources of evidence that the data support the model presented in the Section 5 we fitted models using linear and quadratic time trends. The value of the GPL for the model fitted by using the linear trend was -4572.300 . Similarly, the value of the GPL for the model fitted by using the quadratic trend was -4477.670 . Thus, we have clear evidences that the model presented in the Section 5 provides the best fit among the polynomial alternatives considered to describe the time trend. Furthermore, the same conclusion is obtained when penalizing the Gaussian pseudo log-likelihood with penalties compatible with the Akaike and Kullback-Leibler information criterion (Bonat; 2016).

\section{Concluding remarks}

We presented a flexible class of multivariate models for handling count data. The models were motivated by a data set consisting of the number of blue duikers and other small animals shot or snared by 52 commercial hunters in Bioko Island, Equatorial Guinea. The analysis of the data showed interesting features as overdispersion, excess of zeroes and negatively correlated response variables, which in turn allowed to show the flexibility of our models.

In our framework overdispersion and excess of zeroes are taken into account by 
means of a dispersion function. It is similar to a variance function in the context of generalized linear models. The dispersion function allows to specify models based only on second-moment assumptions and adopts an estimating function approach for parameter estimation and inference. The advantage of the estimating function approach is that the estimation procedure relies on a simple and efficient Newton scoring algorithm. In this paper, we adopted the dispersion function associated with the Poisson-Tweedie distribution, since important discrete distributions as the Neyman Type A, negative binomial and Poisson-inverse Gaussian appear as special cases.

The marginal covariance structure within response variables is specified by means of a matrix linear predictor composed of known matrices. This specification easily deals with the combination of unbalanced repeated measures and longitudinal structures as well as the effects of the covariates in a linear mixed model fashion. The flexibility of this structure comes with the issue to select its components. In this paper, we extended the SIC to guide the selection of the matrix linear predictor components. The great advantage of the SIC is its simplicity. Since the SIC is based on the score statistics it can be computed without actually fitting all the candidate models.

The strategy employed in this paper for selecting the components of the linear and matrix linear predictors consisted of combining the SIC and Wald statistics in a stepwise procedure applied independently for the mean and covariance structures. In the first step, we selected the components of the linear predictor for each response variable assuming independent observations. In fact, in this step we are purposely ignoring the correlation between and within response variables. It is well known that in the presence of correlation the standard errors associated with the regression parameters are underestimated. In this way, we avoid to remove important covariates of the analysis. In the second step, we fixed the linear predictor as obtained in the first step and selected the components of the matrix linear predictor. As the linear predictor potentially contains all significant covariates, we avoid that missing covariates affect the selection of the matrix linear predictor components. In the last step, we fit the multivariate model and remove any non-significant effect.

Finally, the joint covariance matrix is specified by using the generalized Kronecker product. This specification combined with the possibility to estimate the power parameter for each marginal response variable allow our models easily deal with negatively correlated and unequal marginal response variables, overcoming the main limitations of the multivariate Poisson and negative binomial models.

The main limitation of the models presented in this paper is the general lack of algorithms for simulation. Recent work of Baccini et al. (2015) discussed the prob- 
lems involving the simulation of univariate Poisson-Tweedie distributions. The related topic of simulation of the multivariate Tweedie distributions was addressed recently by Cuenin et al. (2015), but the extension to multivariate Poisson-Tweedie distributions specified by general covariance structures in high dimension, as used in this paper, still requires further theoretical and computational developments.

\section{Supplement material}

Dataset and $\mathrm{R}$ code for the analysis are available at the paper companion page at http ://www _ l eg_ufpr_br/doku_php/publications : papercompanions : hunting bioko2016. The authors thank Professors Elias Teixeira Krainski, Walmes Marques Zeviani, Fernando Poul Mayer and Paulo Justianiano Ribeiro Jr for their comments and suggestions that substantially improve the article. The first author is supported by CAPES (Coordenação de Aperfeiçoamento de Pessoal de Nível Superior)-Brazil.

\section{References}

Anderlucci, L. and Viroli, C. (2015). Covariance pattern mixture models for the analysis of multivariate heterogeneous longitudinal data, The Annals of Applied Statistics 9(2): 777-800.

Anderson, T. W. (1973). Asymptotically efficient estimation of covariance matrices with linear structure, The Annals of Statistics 1(1): 135-141.

Baccini, A., Barabesi, L. and Stracqualursi, L. (2015). Random variate generation and connected computational issues for the Poisson-Tweedie distribution, Computational Statistics pp. 1-20. in press.

Bonat, W. H. (2016). mcglm: Multivariate Covariance Generalized Linear Models. $\mathrm{R}$ package version 0.3.0.

URL: https://github.com/wbonat/mcglm

Bonat, W. H. and Jørgensen, B. (2016). Multivariate covariance generalized linear models, Journal of the Royal Statistical Society: Series C (Applied Statistics) . to appear.

Bonat, W. H., Jørgensen, B., Kokonendji, C. C., Hinde, J. and Démetrio, C. G. B. (2016). Extended Poisson-Tweedie: properties and regression models for count data, ArXiv . to appear. 
Breslow, N. E. and Clayton, D. G. (1993). Approximate inference in generalized linear mixed models, Journal of the American Statistical Association 88(421): 925.

Carey, V. J. and Wang, Y. (2011). Working covariance model selection for generalized estimating equations, Statistics in Medicine 30(26): 3117-3124.

Cuenin, J., Jørgensen, B. and Kokonendji, C. C. (2015). Simulations of full multivariate Tweedie with flexible dependence structure, Computational Statistics pp. 1-16. in press.

Cybis, G. B., Sinsheimer, J. S., Bedford, T., Mather, A. E., Lemey, P. and Suchard, M. A. (2015). Assessing phenotypic correlation through the multivariate phylogenetic latent liability model, The Annals of Applied Statistics 9(2): 969-991.

Demidenko, E. (2013). Mixed Models: Theory and Applications with R, Wiley.

Diggle, P. J., Heagerty, P., Liang, K.-Y. and Zeger, S. L. (2002). Analysis of Longitudinal Data, Oxford Statistical Science Series, Oxford.

Fa, J. E. and Brown, D. (2009). Impacts of hunting on mammals in African tropical moist forests: a review and synthesis, Mammal Review 39(4): 231-264.

Fa, J. E., Yuste, J. E. G. and Castelo, R. (2000). Bushmeat markets on Bioko Island as a measure of hunting pressure, Conservation Biology 14(6): 1602-1613.

Fong, Y., Rue, H. and Wakefield, J. (2010). Bayesian inference for generalized linear mixed models, Biostatistics 11(3): 397-412.

Grande-Vega, M., Farfán, M. Á., Ondo, A. and Fa, J. E. (2015). Decline in hunter offtake of blue duikers in Bioko Island, Equatorial Guinea, African Journal of Ecology .

Højsgaard, S., Halekoh, U. and Yan, J. (2006). The R package geepack for Generalized Estimating Equations, Journal of Statistical Software 15(2): 1-11.

Hui, F. K. C., Warton, D. I. and Foster, S. D. (2015). Multi-species distribution modeling using penalized mixture of regressions, Ann. Appl. Stat. 9(2): 866-882.

Jørgensen, B. (1997). The Theory of Dispersion Models, Chapman \& Hall, London.

Jørgensen, B. and Kokonendji, C. (2016). Discrete dispersion models and their Tweedie asymptotics, AStA Advances in Statistical Analysis 100(1): 43-78. 
Klein, N., Kneib, T., Klasen, S. and Lang, S. (2015). Bayesian structured additive distributional regression for multivariate responses, Journal of the Royal Statistical Society: Series C (Applied Statistics) 64(4): 569-591.

Klein, N., Kneib, T., Lang, S. and Sohn, A. (2015). Bayesian structured additive distributional regression with an application to regional income inequality in Germany, The Annals of Applied Statistics 9(2): 1024-1052.

Lagona, F., Maruotti, A. and Padovano, F. (2015). Multilevel multivariate modelling of legislative count data, with a hidden markov chain, Journal of the Royal Statistical Society: Series A (Statistics in Society) 178(3): 705-723.

Lee, Y. and Nelder, J. A. (1996). Hierarchical generalized linear models, Journal of the Royal Statistical Society. Series B (Methodological) 58(4): 619-678.

Liang, K.-Y. and Zeger, S. L. (1986). Longitudinal data analysis using generalized linear models, Biometrika 73(1): 13-22.

Manrique-Vallier, D. (2014). Longitudinal mixed membership trajectory models for disability survey data, The Annals of Applied Statistics 8(4): 2268-2291.

Martinez-Beneito, M. A. (2013). A general modelling framework for multivariate disease mapping, Biometrika 100(3): 539-553.

Masarotto, G. and Varin, C. (2012). Gaussian copula marginal regression, Electronic Journal of Statistics 6: 1517-1549.

McCulloch, C. E. (1997). Maximum likelihood algorithms for generalized linear mixed models, Journal of the American Statistical Association 92(437): 162-170.

Ovaskainen, O. and Soininen, J. (2011). Making more out of sparse data: hierarchical modeling of species communities, Ecology 92(2): 289-295.

Pourahmadi, M. (2000). Maximum likelihood estimation of generalised linear models for multivariate normal covariance matrix, Biometrika 87(2): 425-435.

R Core Team (2015). R: A Language and Environment for Statistical Computing, R Foundation for Statistical Computing, Vienna, Austria. ISBN 3-900051-07-0.

Rodrigues-Motta, M., Pinheiro, H. P., Martins, E. G., Araújo, M. S. and dos Reis, S. F. (2013). Multivariate models for correlated count data, Journal of Applied Statistics 40(7): 1586-1596. 
Shi, P. and Valdez, E. A. (2014). Multivariate negative binomial models for insurance claim counts, Insurance: Mathematics and Economics 55(2014): 18-29.

Stoklosa, J., Gibb, H. and Warton, D. I. (2014). Fast forward selection for generalized estimating equations with a large number of predictor variables, Biometrics 70(1): 110-120.

Tsionas, E. G. (1999). Bayesian analysis of the multivariate Poisson distribution, Communications in Statistics-Theory and Methods 28(2): 431-451.

Verbeke, G., Fieuws, S., Molenberghs, G. and Davidian, M. (2014). The analysis of multivariate longitudinal data: A review, Statistical Methods in Medical Research 23(1): 42-59. 